\title{
Time-Dependent Density-Functional Theory beyond the Local-Density Approximation
}

\author{
Jianmin Tao and Giovanni Vignale \\ Department of Physics, University of Missouri-Columbia, Columbia, Missouri 65211, USA
}

(Received 17 April 2006; published 19 July 2006)

\begin{abstract}
Approximations for the ground-state exchange-correlation potential of density-functional theory have reached a high level of sophistication. By contrast, time- or frequency-dependent exchange-correlation potentials are still being treated in a local approximation. Here we propose a novel approximation scheme, which effectively brings the power of the generalized gradient approximation (GGA) and meta-GGA to time-dependent density-functional theory. The theory should allow a more accurate treatment of strongly inhomogeneous electronic systems (e.g. molecular junctions) while remaining essentially exact for slowly varying densities and slowly varying external potentials.
\end{abstract}

Time-dependent density-functional theory (DFT) $[1,2]$ has become a popular tool for the investigation of the dynamics of many-electron systems. This theory treats the interacting electrons in a time-dependent external potential $V_{\text {ext }}(\mathbf{r}, t)$ as noninteracting electrons moving in a time-dependent effective potential $V_{\text {ext }}(\mathbf{r}, t)+U_{\mathrm{H}}(\mathbf{r}, t)+$ $V_{\mathrm{xc}}(\mathbf{r}, t)$, where $U_{\mathrm{H}}$ is the Hartree potential and $V_{\mathrm{xc}}$ the exchange-correlation (XC) potential. In principle, only the $\mathrm{XC}$ potential remains to be approximated. The simplest approximation is the adiabatic approximation [3], which employs the same form of the XC potential as the static theory but replaces the equilibrium density $n_{0}(\mathbf{r})$ with the instantaneous density $n(\mathbf{r}, t)$. While the adiabatic approximation works well for single-particle excitations of atoms and molecules, it fails for two- and more-particle excitations or charge transfer and gives no dissipation. Furthermore, it severely underestimates the dielectric polarizability of insulators, in spite of the fact that this is a "zero-frequency property." Early attempts [4] to correct the adiabatic approximation without leaving the framework of the local-density approximation were found [5,6] to violate the "harmonic potential theorem." The fundamental reason [7] for the failure of these attempts is that the dynamical XC potential is a fully nonlocal functional in space, and, thus, a consistent local-density approximation from a gradient expansion of the functional does not exist.

Vignale and Kohn (VK) [7] pointed out that a dynamical local-density approximation is still permissible if one switches the basic variable from the density to the current density $\mathbf{j}(\mathbf{r}, t)$. Starting from this observation, they went on to develop the time-dependent current density-functional theory in the linear response regime. However, the VK theory relies on a double local-density approximation (double LDA), namely, both the ground-state density and the external potential are assumed to be slowly varying in space. Under these assumptions, the XC "electric field" $\mathbf{E}_{\mathrm{xc}}(\mathbf{r}, \omega)$ can be expressed in the hydrodynamical form [8]

$$
e E_{\mathrm{xc}, \mu}=\partial_{\mu} V_{\mathrm{xc}}^{\mathrm{ALDA}}(\mathbf{r}, \omega)+\frac{1}{n_{0}} \partial_{\nu} \Delta P_{\mu \nu}^{h, \mathrm{xc}}(\mathbf{r}, \omega)
$$

where $e$ is the absolute value of the electron charge, $V_{\mathrm{xc}}^{\mathrm{ALDA}}$ is the adiabatic local-density approximation (ALDA), and $\Delta P_{\mu \nu}^{h, \mathrm{xc}}$ is the dynamical $\mathrm{XC}$ stress tensor of the homogeneous $(h)$ electron liquid (evaluated at the local density), which gives the linear-order correction to the ALDA. Here and in the following, $\mu, \nu$ are Cartesian indices, $\partial_{\mu}$ is the derivative with respect to $r_{\mu}$, and repeated indices are summed over. The precise statement of the double LDA is that both the wave vector $k$ of the external field and the wave vector $q \sim\left|\nabla n_{0} / n_{0}\right|$, which characterizes the rate of spatial variation of the ground-state density, must be small in comparison with the local Fermi wave vector $k_{F}$ and with $\omega / v_{F}$, where $\omega$ is the frequency of the external field and $v_{F}$ is the Fermi velocity. In short, $q, k \ll \omega / v_{F}, k_{F}$. While these conditions may be satisfied by typical solids, the conditions on $q$ are likely to be violated by molecules, particularly at low frequency. Thus, a difficulty arises when VK is employed to treat systems that have moderately or strongly inhomogeneous densities and are exposed to lowfrequency fields, as, for example, in molecular transport problems [9]. Recent tests on molecules [10], conjugated polymers [11], and quantum wells [12] have shown that Eq. (1) works well for systems with free-electron-like features, or for properties involving only delocalized orbitals, but not for those involving localized orbitals.

The aim of this Letter is to present a method to avoid one of the two local-density approximations on which the VK theory is based, namely, the approximation that the groundstate density is slowly varying. The basic idea is to describe the time evolution of the system in a noninertial reference frame - the Lagrangian frame or $L$ frame - in which the particle density is time-independent and the macroscopic current vanishes [13]. The advantage of working in this reference frame is that the extreme nonlocality that plagues the time-dependent $\mathrm{XC}$ functional in the laboratory frame is cured. So, while the Hamiltonian in the $L$ frame is still time-dependent and inhomogeneous, the fact that the particles in this frame cannot move in or out of a given volume element enables us to apply the powerful technology of the generalized gradient approximation (GGA) [14] and meta- 
GGA [15] to the calculation of the XC stress tensor. In particular, after separating out the exact adiabatic XC potential, we find that the $\mathrm{XC}$ stress tensor has a purely elastic (nondissipative) component, which can be expressed exactly in terms of the ground-state kinetic and potential energy functionals. These functionals can be accurately approximated by a GGA or meta-GGA.

The logic of our approach is as follows. First, we calculate the XC stress tensor $\tilde{P}_{\mu \nu}^{\mathrm{xc}}(\xi, t)$ in the $L$ frame. Then we transform it back to the laboratory frame and subtract from it the adiabatic approximation: This gives the dynamical stress tensor $\Delta P_{\mu \nu}^{\mathrm{xc}}$ of the inhomogeneous system. Finally, the $\mathrm{XC}$ field is calculated from the expression

$$
e E_{\mathrm{xc}, \mu}=\partial_{\mu} V_{\mathrm{xc}}^{\mathrm{ad}}(\mathbf{r}, \omega)+\frac{1}{n_{0}} \partial_{\nu} \Delta P_{\mu \nu}^{\mathrm{xc}}(\mathbf{r}, \omega),
$$

where $V_{\mathrm{xc}}^{\mathrm{ad}}$ is the "exact" adiabatic approximation (i.e., in practice, an adiabatic approximation that includes gradient corrections beyond the ALDA).

The two conditions for the validity of this treatment are (i) the deformation of the ground-state density must be small, and (ii) it must be slowly varying in space, so that its spatial derivative can be neglected. These conditions are more easily met than the condition of slowly varying density. Therefore, our dynamical XC potential should be valid in a wider class of systems than the VK potential, while reducing to the latter in the limit in which the equilibrium density is slowly varying.

The formulation of quantum many-body dynamics in the Lagrangian reference frame has been recently described by Tokatly [13], and we will make use here of some of his results. Let $\mathbf{r}(t)$ denote the position of a small volume element of the electron liquid at time $t$ in the laboratory frame, and let $\boldsymbol{\xi}$ be the position of the same volume element at time $t=0$. The one-to-one correspondence between $\mathbf{r}(t)$ and $\boldsymbol{\xi}$ allows us to define the $L$ frame, in which the position of the volume element is determined by the time-independent vector $\boldsymbol{\xi}$. We can then write

$$
\mathbf{r}(t)=\boldsymbol{\xi}+\mathbf{u}(\mathbf{r}, t)
$$

where $\mathbf{u}$ is the elastic displacement field (notice that in the linear approximation it makes no difference whether $\mathbf{u}$ is considered a function of $\mathbf{r}$ or of $\boldsymbol{\xi}$ ).

According to Ref. [13], the Hamiltonian in the $L$ frame has the form

$$
\hat{\tilde{H}}(t)=\hat{H}+\frac{1}{2} \hat{P}_{\mu \nu}^{\mathrm{eq}} u_{\mu \nu}(t)+\hat{U}_{\mathrm{sc}}(\boldsymbol{\xi}, t),
$$

where $\hat{H}$ is the usual time-independent many-body Hamiltonian of the inhomogeneous system, $\hat{P}_{\mu \nu}^{\mathrm{eq}}$ is the stress-tensor operator $[13,16]$ in the unperturbed ground state, and $u_{\mu \nu} \equiv-\partial_{\nu} u_{\mu}-\partial_{\mu} u_{\nu}$ is the negative of the strain tensor of classical elasticity theory - a direct measure of the deformation of the ground-state density. $\hat{U}_{\mathrm{sc}}(\boldsymbol{\xi}, t)$ is an effective time-dependent potential, which must be determined self-consistently with $\tilde{P}_{\mu \nu}(\xi, t)$. Its presence ensures the constancy of the density and the vanishing of the current.

The stress tensor in the $L$ frame (at frequency $\omega$ ) is the sum of two terms:

$$
\tilde{P}_{\mu \nu}(\boldsymbol{\xi}, \omega)=P_{\mu \nu}^{\mathrm{eq}}(\boldsymbol{\xi})+\int d \xi^{\prime} \tilde{Q}_{\mu \nu \alpha \beta} u_{\alpha \beta}\left(\boldsymbol{\xi}^{\prime}, \omega\right),
$$

where $\tilde{Q}_{\mu \nu \alpha \beta}=\tilde{Q}_{\mu \nu \alpha \beta}^{\infty}\left(\boldsymbol{\xi}, \boldsymbol{\xi}^{\prime}\right)+\Delta \tilde{Q}_{\mu \nu \alpha \beta}\left(\boldsymbol{\xi}, \boldsymbol{\xi}^{\prime}, \omega\right)$ is the elasticity tensor. The first term on the right-hand side of Eq. (5) is just the equilibrium stress tensor, calculated in the ground state of $\hat{H} . \tilde{Q}_{\mu \nu \alpha \beta}^{\infty}\left(\boldsymbol{\xi}, \boldsymbol{\xi}^{\prime}\right)$ is the frequencyindependent (instantaneous in time) component of the elasticity tensor. It is given by the derivative of the stress tensor $\tilde{P}_{\mu \nu}(\xi, \omega)$ with respect to the strain tensor $u_{\alpha \beta}$, evaluated at the ground state of $\hat{H}$. Finally, the $\Delta \tilde{Q}_{\mu \nu \alpha \beta}$ term, which in general is a complex function of frequency, represents the change in the expectation value of the stresstensor operator due to the fact that the system is not in the ground state but evolves under the action of $\hat{\tilde{H}}(t)$. This term is crucial for dissipation (see discussion below) and leads to a renormalization of the elastic constants at finite frequency. However, it vanishes in the high-frequency limit: so the high-frequency elastic response is entirely controlled by $\tilde{Q}_{\mu \nu \alpha \beta}^{\infty}$ (hence the superscript $\infty$ ).

The XC part of the stress tensor is obtained by repeating the above procedure for a noninteracting system with the same equilibrium density and subtracting the noninteracting stress tensor from the interacting one [Eq. (5)]. Then we calculate the XC elasticity tensor $\tilde{Q}_{\mu \nu \alpha \beta}^{\mathrm{xc} \infty}\left(\boldsymbol{\xi}, \boldsymbol{\xi}^{\prime}\right)$, under the assumptions that the deformation $u_{\mu \nu}(\mathbf{r}, \omega)$ is small and slowly varying in space. This very complicated $\mathrm{XC}$ elasticity tensor ( 21 independent components, in general) is greatly simplified by the further assumption that the pair correlation function $g\left(\boldsymbol{\xi}, \boldsymbol{\xi}^{\prime}\right)$ and the correlation part of the one-particle density matrix $\rho_{1}\left(\boldsymbol{\xi}, \boldsymbol{\xi}^{\prime}\right)$ have spherical symmetry about any fixed point $\boldsymbol{\xi}$. This "local isotropy" assumption underlies the successful formulations of the GGA and meta-GGA holes [17]. With this assumption, we are able to represent the fourth-rank XC elasticity tensor with only two quantities $\tilde{Q}_{\alpha \alpha \beta \beta}^{\mathrm{xc}}$ and $\tilde{Q}_{\alpha \beta \alpha \beta}^{\mathrm{xc}}$, which are related to a local bulk modulus and a local shear modulus, respectively. More precisely, we have

$$
\begin{aligned}
& \tilde{Q}_{\alpha \alpha \beta \beta}^{\mathrm{xc} \infty}=\left[3 T_{\mathrm{c}}\left(\left[n_{0}\right] ; \boldsymbol{\xi}\right)+V_{\mathrm{ee}}^{\mathrm{xc}}\left(\left[n_{0}\right] ; \boldsymbol{\xi}\right)\right] \delta\left(\boldsymbol{\xi}-\boldsymbol{\xi}^{\prime}\right), \\
& \tilde{Q}_{\alpha \beta \alpha \beta}^{\mathrm{xc \infty}}=\left[T_{\mathrm{c}}\left(\left[n_{0}\right] ; \boldsymbol{\xi}\right)-2 V_{\mathrm{ee}}^{\mathrm{xc}}\left(\left[n_{0}\right] ; \boldsymbol{\xi}\right)\right] \delta\left(\boldsymbol{\xi}-\boldsymbol{\xi}^{\prime}\right),
\end{aligned}
$$

where $T_{\mathrm{c}}\left(\left[n_{0}\right] ; \boldsymbol{\xi}\right)$ and $V_{\mathrm{ee}}^{\mathrm{xc}}\left(\left[n_{0}\right] ; \boldsymbol{\xi}\right)$ are, respectively, the kinetic correlation and XC potential energy densities in the ground state. Furthermore, we have $P_{\mu \nu}^{\mathrm{xc}}=\frac{1}{3} \times$ $\left[2 T_{\mathrm{c}}\left(\left[n_{0}\right] ; \boldsymbol{\xi}\right)+V_{\mathrm{ee}}^{\mathrm{xc}}\left(\left[n_{0}\right] ; \boldsymbol{\xi}\right)\right] \delta_{\mu \nu}$, which is a direct consequence of the virial theorem.

We now transform the XC stress tensor of Eq. (5) back to the laboratory frame and subtract the part that pertains to the adiabatic approximation, namely, $P_{\mu \nu}^{\mathrm{xc} \text { ad }}(\mathbf{r}, t)=$ $\delta_{\mu \nu} P^{\mathrm{xc} \text { eq }}([n] ; \mathbf{r}, t)$, where $n=n_{0}-\nabla \cdot\left(n_{0} \mathbf{u}\right)$. Applying the transformation rule for tensors [13], we find that the 
first-order correction to the adiabatic XC stress tensor in the laboratory frame is given by

$$
\begin{aligned}
\Delta P_{\mu \nu}^{\mathrm{xc}}(\mathbf{r}, \omega)= & {\left[Q_{\mu \nu \alpha \beta}^{\mathrm{xc} \infty}(\mathbf{r})+\Delta \tilde{Q}_{\mu \nu \alpha \beta}^{\mathrm{xc}}(\mathbf{r}, \omega)\right] u_{\alpha \beta} } \\
& -\frac{\delta_{\mu \nu}}{2} K_{\mathrm{xc}}^{0}(\mathbf{r}) u_{\alpha \alpha},
\end{aligned}
$$

where $K_{\mathrm{xc}}^{0}(\mathbf{r})$ is the zero-frequency limit of the XC bulk modulus defined by

$$
K_{\mathrm{xc}}^{0}(\mathbf{r})=\int d^{3} r^{\prime} n_{0}\left(\mathbf{r}^{\prime}\right) \frac{\delta P^{\mathrm{xc}}\left(\left[n_{0}\right] ; \mathbf{r}\right)}{\delta n_{0}\left(\mathbf{r}^{\prime}\right)},
$$

with $P^{\mathrm{xc}}=\left(2 T_{\mathrm{c}}+V_{\mathrm{ee}}^{\mathrm{xc}}\right) / 3$ being the local XC pressure and $Q_{\mu \nu \alpha \beta}^{\mathrm{xc} \infty}=\frac{1}{2} P^{\mathrm{xc} \mathrm{eq}}\left(\left[n_{0}\right] ; \mathbf{r}\right)\left(\delta_{\mu \alpha} \delta_{\nu \beta}+\delta_{\nu \alpha} \delta_{\mu \beta}\right)+\tilde{Q}_{\mu \nu \alpha \beta}^{\mathrm{xc} \infty}$.

According to the local isotropy assumption, the elasticity tensor in the laboratory frame can be represented as

$$
\begin{aligned}
Q_{\mu \nu \alpha \beta}^{\mathrm{xc}}(\mathbf{r}, \omega)= & \frac{K_{\mathrm{xc}}(\mathbf{r}, \omega)}{2} \delta_{\mu \nu} \delta_{\alpha \beta}+\mu_{\mathrm{xc}}(\mathbf{r}, \omega) \\
& \times\left(-\frac{\delta_{\mu \nu} \delta_{\alpha \beta}}{3}+\frac{\delta_{\mu \alpha} \delta_{\nu \beta}+\delta_{\nu \alpha} \delta_{\mu \beta}}{2}\right),
\end{aligned}
$$

where $Q_{\mu \nu \alpha \beta}^{\mathrm{xc}}=Q_{\mu \nu \alpha \beta}^{\mathrm{xc} \infty}+\Delta \tilde{Q}_{\mu \nu \alpha \beta}^{\mathrm{xc}}$, and $K_{\mathrm{xc}}$ and $\mu_{\mathrm{xc}}$ are the complex XC bulk and shear moduli [18], respectively. Substituting Eq. (9) into Eq. (7) yields the linear-order correction to the exact adiabatic XC stress tensor

$$
\begin{aligned}
\Delta P_{\mu \nu}^{\mathrm{xc}}(\mathbf{r}, \omega)= & \frac{\delta_{\mu \nu}}{2}\left[K_{\mathrm{xc}}(\mathbf{r}, \omega)-K_{\mathrm{xc}}^{0}(\mathbf{r})\right] u_{\alpha \alpha} \\
& +\mu_{\mathrm{xc}}(\mathbf{r}, \omega)\left(u_{\mu \nu}-\frac{\delta_{\mu \nu}}{3} u_{\alpha \alpha}\right) .
\end{aligned}
$$

In the high-frequency limit, making use of Eq. (6) we find

$$
\begin{aligned}
& K_{\mathrm{xc}}^{\infty}=\frac{10}{9} T_{\mathrm{c}}\left(\left[n_{0}\right] ; \mathbf{r}\right)+\frac{4}{9} V_{\mathrm{ee}}^{\mathrm{xc}}\left(\left[n_{0}\right] ; \mathbf{r}\right), \\
& \mu_{\mathrm{xc}}^{\infty}=\frac{2}{3} T_{\mathrm{c}}\left(\left[n_{0}\right] ; \mathbf{r}\right)-\frac{2}{15} V_{\mathrm{ee}}^{\mathrm{xc}}\left(\left[n_{0}\right] ; \mathbf{r}\right) .
\end{aligned}
$$

In practical applications, $T_{\mathrm{c}}\left(\left[n_{0}\right] ; \mathbf{r}\right)$ and $V_{\mathrm{ee}}^{\mathrm{xc}}\left(\left[n_{0}\right] ; \mathbf{r}\right)$ may be approximated as in the static DFT. The first three rungs of a ladder of nonempirical density functionals [19] for ground states have been constructed. In particular, the meta-GGA [15] has achieved uniform accuracy in both condensed matter physics and quantum chemistry [20] and has been extended [21] to describe systems in a magnetic field. Within LDA, GGA, or meta-GGA, the kinetic energy density of correlation is given by [22]

$$
T_{\mathrm{c}}\left(\left[n_{0}\right] ; \mathbf{r}\right)=-n_{0} \frac{\partial}{\partial r_{s}}\left[r_{s} \epsilon_{\mathrm{c}}\left(r_{s}, s_{1}, s_{2}, \cdots\right)\right],
$$

where $r_{s}(\mathbf{r})=a_{0}^{-1}\left(3 / 4 \pi n_{0}\right)^{1 / 3}, s_{1}, s_{2} \ldots$ are dimensionless variables that do not depend upon $a_{0}$ (such as $s_{1}=$ $\left.s=\left|\nabla n_{0}\right| / 2 k_{F} n_{0}\right)$, and $\epsilon_{\mathrm{c}}$ is the correlation energy per electron. The XC potential energy density can be calculated by the relation $V_{\mathrm{ee}}^{\mathrm{xc}}(\mathbf{r})=n_{0} \epsilon_{\mathrm{xc}}-T_{\mathrm{c}}\left(\left[n_{0}\right] ; \mathbf{r}\right)$. This completes our construction of the infinite-frequency part of the XC elastic moduli of Eq. (10).

Finally, let us consider the frequency-dependent parts. They arise from the $\Delta \tilde{Q}_{\mu \nu \alpha \beta}$ term of Eq. (5), as seen from Eq. (9). Because the real parts of these two quantities can be calculated from their imaginary parts with the help of the Kramers-Krönig dispersion relation [2], we need only to calculate the imaginary parts. Even this simplified calculation is, however, very challenging, so we resort to the time-honored practice of interpolating between the highand low-frequency limits of $\operatorname{Im} \Delta \tilde{Q}_{\mu \nu \alpha \beta}[4,23]$.

In the high- $\omega$ limit, second-order perturbation theory $[24,25]$ gives $\operatorname{Im} K_{\mathrm{xc}}=-n_{0}^{2} \pi m e^{4} / 9(m \omega)^{3 / 2}$ and $\operatorname{Im} \mu_{\mathrm{xc}}=$ $-16 n_{0}^{2} \pi m e^{4} / 15(m \omega)^{3 / 2}$. In the low- $\omega$ limit, $\operatorname{Im} K_{\mathrm{xc}}=$ $O\left(\omega^{3}\right)$ and $\operatorname{Im} \mu_{\mathrm{xc}}=-\omega\left(m e^{2} / \pi\right)^{2} k_{F} S_{3}^{T}\left(n_{0}\right)$, where $S_{3}^{T}=$ (3/4) $S_{3}^{L}$ and $S_{3}^{L}$ is given by Eq. (16) of Ref. [23]. $K_{\mathrm{xc}}^{0}$ and $\mu_{\mathrm{xc}}^{0}$ must satisfy the sum rules

$$
M_{\mathrm{xc}}^{0}(\mathbf{r})-M_{\mathrm{xc}}^{\infty}(\mathbf{r})=\int_{-\infty}^{\infty} \frac{d \omega}{\pi} \frac{\operatorname{Im} M_{\mathrm{xc}}(\mathbf{r}, \omega)}{\omega},
$$

where $M=K$ or $\mu$. In order to account for the twoplasmon excitations [25] which occur around $\omega=2 \omega_{p}$, where $\omega_{p}=\sqrt{4 \pi n_{0} e^{2} / m}$ is the plasmon frequency, following Qian and Vignale [23], we require that $\operatorname{Im} K_{\mathrm{xc}}(\mathbf{r}, \omega)$ and $\operatorname{Im} \mu_{\mathrm{xc}}(\mathbf{r}, \omega)$ achieve a minimum around $\omega=2 \omega_{p}$. To satisfy the four conditions, we choose $\operatorname{Im} K_{\mathrm{xc}}(\mathbf{r}, \omega)$ and $\operatorname{Im} \mu_{\mathrm{xc}}(\mathbf{r}, \omega)$ to be of the simplest forms

$$
\begin{aligned}
& \operatorname{Im} \mu_{\mathrm{xc}}=-\omega n_{0} f_{T}(\mathbf{r}, \omega), \\
& \operatorname{Im} K_{\mathrm{xc}}=-\omega n_{0} f_{L}(\mathbf{r}, \omega)-\frac{4}{3} \operatorname{Im} \mu_{\mathrm{xc}},
\end{aligned}
$$

where $f_{L(T)}=a_{L(T)} /\left(1+b_{L(T)} \tilde{\omega}^{2}\right)^{5 / 4}+d_{L(T)} \tilde{\omega}^{2} e^{-\beta \tilde{\omega}^{2}}$, $\beta=3 / 2, \quad a_{L(T)}=n_{0}\left(m e^{2} / n_{0} \pi\right)^{2} k_{F} S_{3}^{L(T)}, \quad$ and $d_{L(T)}=6 \sqrt{3 / 2 \pi}\left[\left(-\pi n_{0} / 4 \omega_{p}\right) \Delta_{L(T)}-\left(\pi \sqrt{2 \pi} / 2\left[\Gamma\left(\frac{1}{4}\right)\right]^{2}\right) \times\right.$ $\left.\left(4 r_{s}^{3} c_{L(T)}^{4} / 3\right)^{1 / 10} a_{L(T)}^{3 / 5}\right]$, with $\quad \tilde{\omega}=\omega / \omega_{p}, \quad b_{L(T)}=$ $16\left[3 a_{L(T)}^{4} /\left(4 r_{s}^{3} c_{L(T)}^{4}\right)\right]^{1 / 5}, c_{L}=23 / 15, c_{T}=16 / 15, \Delta_{T}=$ $\left(\mu_{\mathrm{xc}}^{0}-\mu_{\mathrm{xc}}^{\infty}\right) / n_{0}^{2}$, and $\Delta_{L}=\left(K_{\mathrm{xc}}^{0}-K_{\mathrm{xc}}^{\infty}\right) / n_{0}^{2}+4 \Delta_{T} / 3$. The low- $\omega$ limit of the shear modulus $\mu_{\mathrm{xc}}^{0}$ can be calculated from the random-phase approximation (RPA) [25].

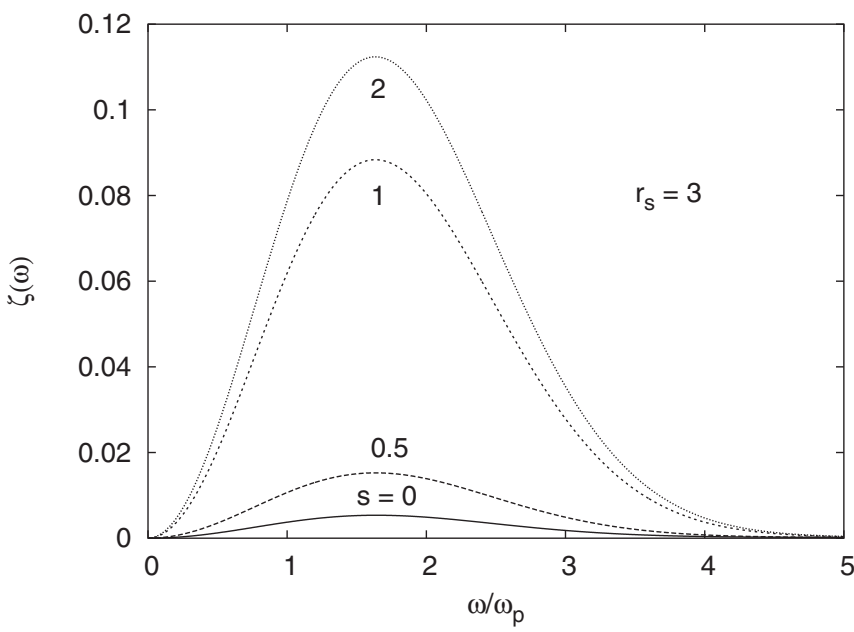

FIG. 1. The bulk viscosity $\zeta(\omega)$ (in units of $\hbar n_{0}$ ) for several values of the reduced gradient $s=\left|\nabla n_{0}\right| / 2 k_{F} n_{0}$ at $r_{s}=3 . s=0$ corresponds to the uniform gas. The same RPA value of $\mu_{\mathrm{xc}}(0)$ for all $s$ is taken from Table I of Ref. [25]. 


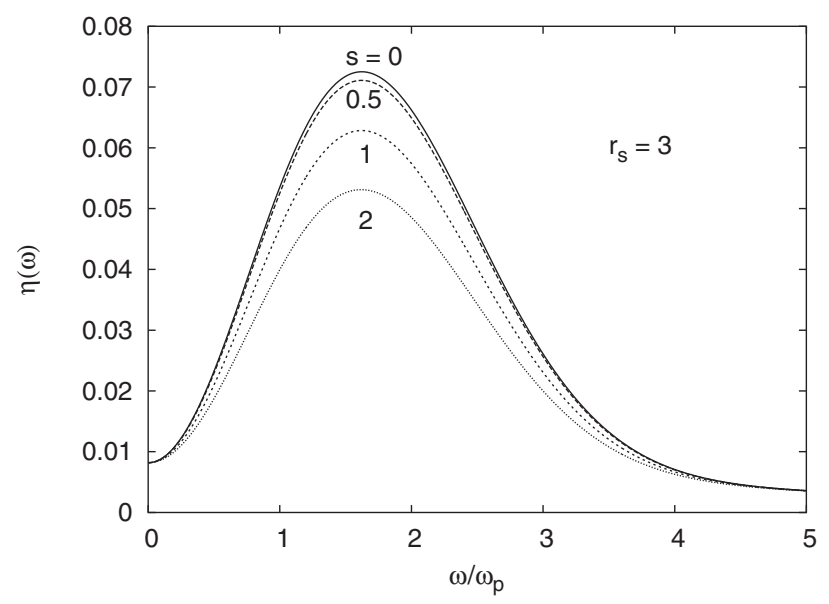

FIG. 2. The shear viscosity $\eta(\omega)$ plotted at $r_{s}=3$. The units and notations are the same as in Fig. 1.

As an example, we calculate $K_{\mathrm{xc}}^{\infty}, K_{\mathrm{xc}}^{0}$, and $\mu_{\mathrm{xc}}^{\infty}$ with the GGA [14] and substitute them into Eq. (14). To show clearly the effect of the inhomogeneity, we assume that the second derivatives of the density are zero, and we plot here the XC bulk viscosity $\zeta(\omega)=-\operatorname{Im} K_{\mathrm{xc}}(\mathbf{r}, \omega) / \omega$ and the shear viscosity $\eta(\omega)=-\operatorname{Im} \mu_{\mathrm{xc}}(\omega) / \omega$ for different values of the reduced density gradient $s$ at a fixed density (see Figs. 1 and 2). Both quantities have direct physical significance [9]. From these figures, we see that the bulk viscosity increases, while the shear viscosity decreases, with increasing $s$, i.e., when the system becomes more inhomogeneous [26]. The dependence on $s$ is very significant and reflects the strong dependence of the high- and low-frequency limits of the elastic moduli. We have calculated the real part of the $\omega$-dependent $\mu_{\mathrm{xc}}$ and found that, like the imaginary part, it decreases with increasing $s$ as well. This may help to improve the description of the dynamical properties of long polymer chains, where the shear modulus (without $s$ dependence) has been found to play an important role [27].

In summary, we have derived the linear-order correction to the exact adiabatic approximation for the dynamical XC potential for an inhomogeneous system. In our derivation, we have assumed that the strain $u_{\alpha \beta}(\mathbf{r})$ is small and slowly varying in space. These conditions do not require the equilibrium density $n_{0}(\mathbf{r})$ to be slowly varying in space. Under these assumptions, the quality of our approximation depends only on how well the GGA and the meta-GGA treat the static inhomogeneous system. In the limit of slowly varying equilibrium density, the $\mathrm{XC}$ stress tensor of Eq. (10) reduces to that of VK and the XC bulk modulus of Eq. (8) reduces to the familiar form $K_{\mathrm{xc}}^{0}=$ $n_{0}^{2} \partial^{2}\left(n_{0} \epsilon_{\mathrm{xc}}\right) / \partial n_{0}^{2}$ as well. The proposed XC potential should improve the description of the dynamic properties of both molecules and solids as well as intermediate systems (e.g. polymers) [27] or combinations thereof (e.g. molecular junctions in transport problems).

We acknowledge valuable discussions with M. Di Ventra and helpful comments of C.A. Ullrich and Robert van Leeuwen. This work was supported by DOE under Grant No. DE-FG02-05ER46203.

[1] E. Runge and E. K. U. Gross, Phys. Rev. Lett. 52, 997 (1984).

[2] G.F. Giuliani and G. Vignale, Quantum Theory of the Electron Liquid (Cambridge University Press, Cambridge, England, 2005).

[3] A. Zangwill and P. Soven, Phys. Rev. Lett. 45, 204 (1980).

[4] E. K. U. Gross and W. Kohn, Phys. Rev. Lett. 55, 2850 (1985).

[5] J. F. Dobson, Phys. Rev. Lett. 73, 2244 (1994).

[6] G. Vignale, Phys. Rev. Lett. 74, 3233 (1995).

[7] G. Vignale and W. Kohn, Phys. Rev. Lett. 77, 2037 (1996); in Electronic Density Functional Theory, edited by J. Dobson, M.P. Das, and G. Vignale (Plenum, New York, 1996).

[8] G. Vignale, C. A. Ullrich, and S. Conti, Phys. Rev. Lett. 79, 4878 (1997). Here $\Delta P_{\mu \nu}^{\mathrm{h}, \mathrm{xc}}=-\sigma_{\mathrm{xc}, \mu \nu}$ of Eq. (4).

[9] Na Sai, M. Zwolak, G. Vignale, and M. Di Ventra, Phys. Rev. Lett. 94, 186810 (2005).

[10] M. van Faassen and P.L. de Boeij, J. Chem. Phys. 120, 8353 (2004).

[11] M. van Faassen et al., Phys. Rev. Lett. 88, 186401 (2002).

[12] C. A. Ullrich and G. Vignale, Phys. Rev. Lett. 87, 037402 (2001).

[13] I. V. Tokatly, Phys. Rev. B 71, 165104 (2005); 71, 165105 (2005).

[14] J. P. Perdew, K. Burke, and M. Ernzerhof, Phys. Rev. Lett. 77, 3865 (1996).

[15] J. Tao, J. P. Perdew, V. N. Staroverov, and G. E. Scuseria, Phys. Rev. Lett. 91, 146401 (2003).

[16] C. L. Rogers and A. M. Rappe, Phys. Rev. B 65, 224117 (2002).

[17] L. A. Constantin, J. P. Perdew, and J. Tao, Phys. Rev. B 73, 205104 (2006).

[18] S. Conti and G. Vignale, Phys. Rev. B 60, 7966 (1999).

[19] J. P. Perdew et al., J. Chem. Phys. 123, 062201 (2005).

[20] V. N. Staroverov et al., J. Chem. Phys. 119, 12129 (2003); 121, 11 507(E) (2004); Phys. Rev. B 69, 075102 (2004).

[21] J. Tao and J.P. Perdew, Phys. Rev. Lett. 95, 196403 (2005).

[22] A. Görling, M. Levy, and J. P. Perdew, Phys. Rev. B 47, 1167 (1993); J. Tao and J. P. Perdew, in Reviews in Modern Quantum Chemistry: A Celebration of the Contributions of R.G. Parr, edited by K.D. Sen (World Scientific, Singapore, 2002).

[23] Z. Qian and G. Vignale, Phys. Rev. B 65, 235121 (2002).

[24] A. J. Glick and W. F. Long, Phys. Rev. B 4, 3455 (1971).

[25] R. Nifosí, S. Conti, and M. P. Tosi, Phys. Rev. B 58, 12758 (1998).

[26] However, this trend is reversed in the large-gradient $(s \rightarrow$ $\infty$ ) limit. In this limit, the exchange part of the GGA (and meta-GGA) takes the same form as in the uniform gas, but multiplied by 1.804 , while the correlation part vanishes [14]: Therefore, the viscosity spectra tend to the constants independent of $s$.

[27] J. A. Berger, P.L. de Boeij, and R. van Leeuwen, Phys. Rev. B 71, 155104 (2005). 